\title{
MRI Changes with Acute Shingles
}

\author{
Kathleen Joy Khu, Mark Bernstein
}

Can. J. Neurol. Sci. 2009; 36: 787-788

We describe magnetic resonance imaging (MRI) changes in the brain parenchyma and tentorium ipsilateral to the involved trigeminal nerve associated with acute herpes zoster ophthalmicus infection. To our knowledge, only one other similar case has been reported.

\section{Case Report}

A 68-year-old lady presented with low-grade fever, malaise, and flu-like symptoms and a week later noted a painful rash over the left side of her scalp. She had a past history of a left frontal parasagittal meningioma excised two years previously, and was scheduled for a routine follow-up MRI around this time, when the patient was symptomatic. On examination the skin over the left forehead in the V1 distribution of the left trigeminal nerve was erythematous and covered with vesicles. There were no changes in visual acuity, and no other neurologic deficits. She was diagnosed to have herpes zoster (shingles) and was immediately started on an oral anti-viral agent with resolution of the rash and periorbital swelling after several days, although she still complained of pain.

An MRI was done five days after the appearance of the rash but prior to treatment, as part of the routine follow-up imaging for the meningioma. It showed enhancement of the left tentorium on $\mathrm{T} 1$, as well as an area of hyperintensity on the tentorium and the brain parenchyma overlying it, which corresponds to the inferior medial aspect of the left temporo-occipital region, on the fluid-attenuated inversion-recovery (FLAIR) sequence (Figure 1). These changes were not seen on the MRI the previous year, one year following the meningioma surgery. There was some concern that the FLAIR abnormality represented a small cortical venous infarction, thus a repeat MRI with magnetic resonance venography was ordered. The second MRI was done three weeks after the first one, approximately two weeks after the anti-viral treatment was started. The rash had resolved, although the patient still had pain in the V1 distribution. On the repeat MRI, the FLAIR abnormality had completely disappeared (Figure 2), and the MRV was normal. Enhanced coronal T1 enhanced sequences, which showed the dural enhancement most clearly, were not obtained in the second MRI.

\section{DISCUSSION}

Herpes zoster ophthalmicus affects the tissues innervated by the ophthalmic division of the trigeminal nerve. It may cause ocular inflammation and visual loss in addition to pain and skin changes. It accounts for $10-25 \%$ of all cases of shingles, ${ }^{1}$ and is caused by reactivation of the Varicella zoster virus which has hitherto remained latent in the trigeminal ganglion after a primary infection with Varicella.

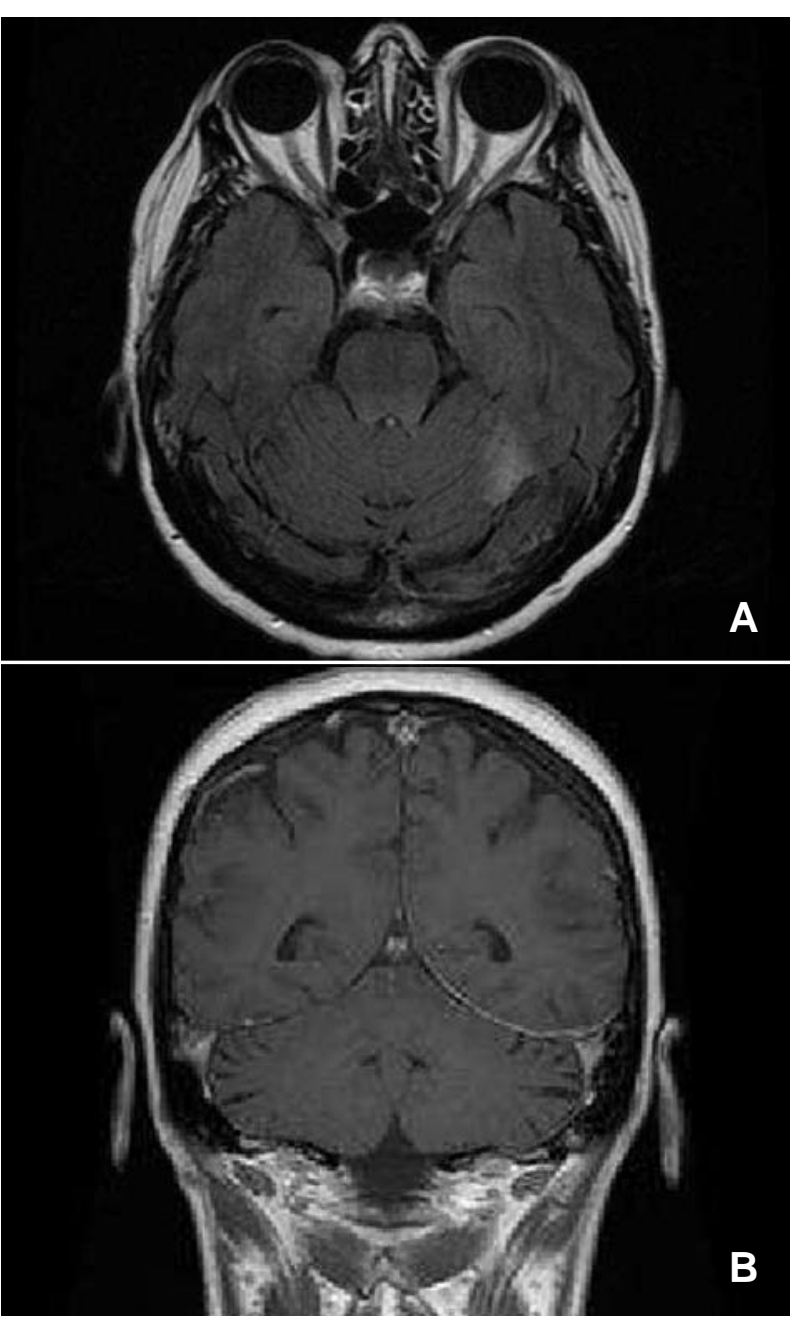

Figure 1: Pre-treatment MRI. Axial T2 FLAIR MRI (A) showing hyperintensity in the left tentorium and the medial temporo-occipital lobe just above it, and coronal T1 gadolinium-enhanced MRI (B) showing enhancement of the left tentorium.
From the Division of Neurosurgery, University of Toronto, Toronto Western Hospital, Toronto, Ontario, Canada.

Received April 21, 2009. Final Revisions Submitted May 28, 2009. Correspondence to: Mark Bernstein, Division of Neurosurgery, University of Toronto, Toronto Western Hospital, 399 Bathurst Street, 4 West Wing, Toronto, Ontario, M5T 2S8, Canada. 
Since herpes zoster is diagnosed primarily by clinical signs and symptoms, there are few imaging data available regarding acute herpes zoster. Haanpaa et $\mathrm{al}^{2}$ noted that 9 out of $16(56 \%)$ patients had focal high signal-intensity areas in the brainstem and spinal cord on T2-weighted scans. Enhancement of the ipsilateral trigeminal nerve was seen in three cases, with concomitant brainstem changes. ${ }^{2}$ Other case reports describe MRI findings of enhancement of the abducens nerve ${ }^{3}$ and enhancement of the oculomotor nerve as well as a high signal on FLAIR sequence within the ipsilateral medulla. ${ }^{4}$ Aribandi et $\mathrm{al}^{5}$ reported increased T2 signal changes in the lower pons, medulla, and upper cervical cord corresponding to the anatomic location of the spinal trigeminal nucleus and tract. These signal changes may represent involvement of the central nervous system parenchyma in the acute stages of herpes zoster, and may be associated with the development of post-herpetic neuralgia. ${ }^{2}$ Some of the MRI lesions resolve completely after institution of treatment, ${ }^{2,3,6}$ whereas some remain unchanged. ${ }^{2}$ The clinical significance of this is uncertain.

Only one other case has been found in the literature with imaging findings similar to our own. Bert et $\mathrm{al}^{7}$ reported the case of an elderly patient who presented with acute herpes zoster ophthalmicus with secondary central nervous system (CNS) spread. Her MRI showed enhancement of the ipsilateral Meckel's cave and tentorium as well as the pons, midbrain, and optic chiasm. There were concomitant FLAIR changes in these parenchymal structures as well. It is believed that these changes represent areas of viral infection and associated inflammation. ${ }^{7}$

Our case illustrates the appearance of MRI signal changes in the inferior temporal-occipital brain parenchyma and tentorium ipsilateral to the involved trigeminal nerve in acute V1 shingles. Most of the MRI signal changes reported in the literature affect the brainstem in trigeminal herpes, implying involvement of the nucleus and/or the sensory ganglion. Involvement of the parenchyma and tentorial dura may suggest direct viral invasion or extension of the inflammation from the affected cranial nerve. This is consistent with one of the postulated routes of spread of herpes zoster to the CNS, which is a transneural spread from the trigeminal ganglion to its innervation of the meninges, followed by cerebrospinal fluid spread to other structures. ${ }^{7}$ The resolution of imaging changes after treatment suggests that the abnormality is indeed related to the herpes zoster infection. One can postulate that seeing MRI changes in herpes zoster patients portends recurrence and/or the development of post-herpetic neuralgia. There is no evidence to confirm this although it is intuitive that pathology substantial enough to effect MRI changes might have more serious and longer-lasting sequelae.

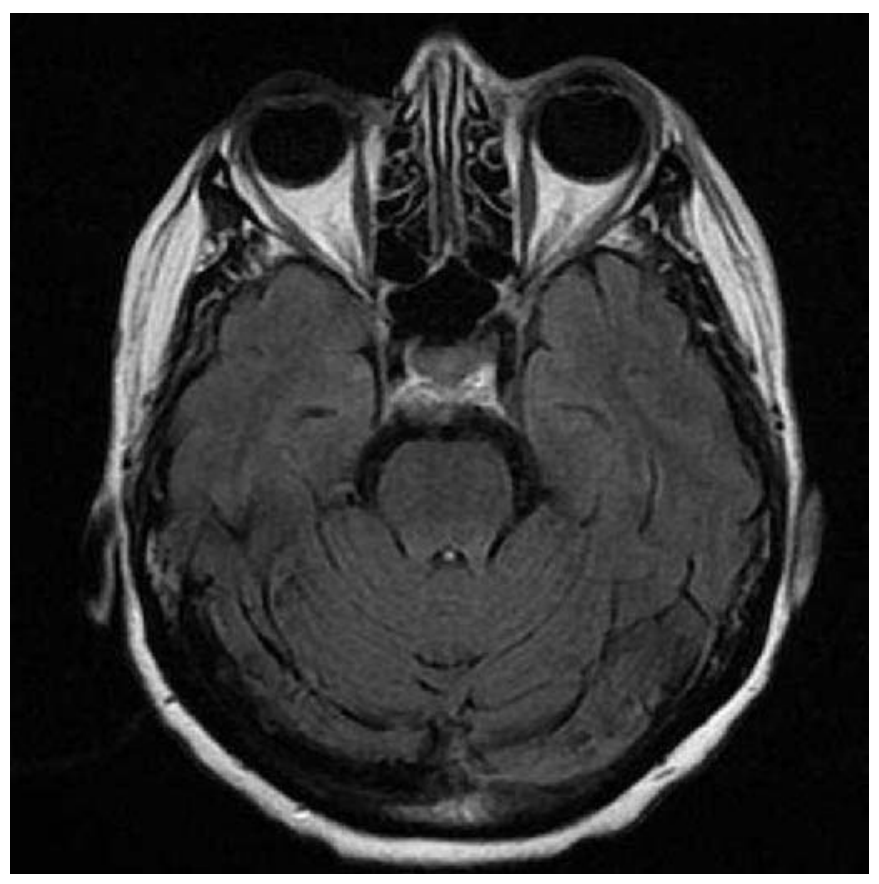

Figure 2: Post-treatment MRI. Axial T2 FLAIR MRI at exact same level as the slice in Figure 1A showing resolution of the signal change.

\section{REFERENCES}

1. Ang LP, Au Eong KG, Ong SG. Herpes zoster ophthalmicus. J Pediatr Ophthalmol Strabismus. 2001;38(3):174-6.

2. Haanpaa M, Dastidar P, Weinberg A, Levin M, Miettinen A, Lapinlampi A, et al. CSF and MRI findings in patients with acute herpes zoster. Neurology. 1998;51(5):1405-11.

3. Keiichiro I, Hiroshi K, Satoshi O. A case of herpes zoster ophthalmicus with abducent palsy: the cause and magnetic resonance imaging findings. J Jap Ophthalmol Soc. 2006;110 (3):193-6

4. Quisling SV, Shah VA, Lee HK, Policeni B, Smoker WR, Martin C, et al. Magnetic resonance imaging of third cranial nerve palsy and trigeminal sensory loss caused by herpes zoster. J Neuroophthalmol. 2006;26(1):47-8.

5. Aribandi M, Aribandi L. MRI of trigeminal zoster. Neurology. 2005;65(11):1812.

6. Otero J, Ribera E, Gavalda J, Rovira A, Ocana I, Pahissa A. Response to acyclovir in two cases of herpes zoster leukoencephalitis and review of the literature. Eur J Clin Microbiol. 1998;17(4):286-9.

7. Bert RJ, Samawareerwa R, Melhem ER. CNS MR and CT findings associated with a clinical presentation of herpetic acute retinal necrosis and herpetic retrobulbar optic neuritis: five HIVinfected and one non-infected patients. AJNR. Am J Neuroradiol. 2004;25 (10): 1722-9. 